\title{
Banana Ripeness Classification using HSV Colour Space and Nearest Centroid Classifier
}

\author{
Elizabeth Nurmiyati Tamatjita *, Rouly Doharma Sihite *
}

\begin{abstract}
Banana is a common fruit which is found throughout Southeast Asia and are beneficial both as delicacy and as a dessert which is good for health. Although quite common and easy to obtain, many people today find it difficult to identify the correct ripeness stage of banana, especially when purchasing from traditional vendors, where varying degree of ripeness are available. This research sought for a possibility to classify banana ripeness by its peel colour with HSV colour space as the feature and classified using Nearest Centroid Classifier (NCC). 'Ambon Lumut', 'Kepok' and 'Raja' bananas are used as examples for the research as they are among the most common types of banana available for many use in Indonesia, and its ripeness is divided into 4 classes according to different usage of the banana: unripe, almost ripe, ripe, and overripe. Photographic images of 'Ambon Lumut', 'Kepok' and 'Raja' bananas are used as training and test data. The experiment is conducted using cleaned images which have the background removed, and this experiment also resulting in $73.33 \%$ recognition. The recognition results for each class respectively are: Green $=93.33 \%$; Almost Ripe $=80 \%$; Ripe $=66.67 \%$ and Overripe $=53.33 \%$.
\end{abstract}

Keywords: Fruit classification, HSV colour space, nearest centroid classifier.

\section{Introduction}

Banana belongs to an array of tropical fruits easily available in Southeast Asia, as well in Indonesia. It has benefits both as a delicacy and as a fruit which is good for health, and it can be enjoyed in many ways: fresh or processed. These different ways to enjoy banana requires it to be at a specific stage of ripeness for every serving: direct consumption, fried, steamed or even processed into powder. Although this may seem as an easy task, it needs experience to correctly determine the stage. Common people might need aid to determine correctly the stages, in order not to waste the banana which was unfit for a specific processing. The ripeness can be indicated by the peel colour of the banana.

This research used a certain type of bananas: the kepok banana (Musa paradisiaca forma typica), which is easily found and can be processed in many ways according to its ripeness, thus provide

\footnotetext{
* PTK - Sekolah Tinggi Manajemen Informatika \& Komputer Widuri, Jakarta, Indonesia
} 
a suitable example for the experiment. It is a common knowledge in Indonesia that kepok banana can be enjoyed fresh when it is ripe, can be fried when it's overripe, can be steamed or made into crisps when it's almost ripe, and even processed into powder when it is still green. Ambon Lumut banana is a local variant of Cavendish banana (Musa acuminata cultivar group AAA) which is often enjoyed fresh as dessert and as a snack. A characteristic of ambon lumut is that during the ripening stage, its peel never turns completely yellow, but only become moss green (thus the name lumut which is moss) and then starts to have brown spots which grow bigger as it overripe. Raja banana can be enjoyed fresh or fried, and aside from its different fruit texture, its peel has the similar colour characteristics as the kepok.

Colour feature of the peel is obtained from RGB values of the image pixels and then converted into HSV colour space in order to emphasize the colour range during the ripeness stage within the hue. The three banana variants is altogether collected as samples for the research. Our hope is that this research will initiate similar researches using other features to further improve the study of fruit ripeness classification.

\section{Related Works}

In order to improve processing, the characterization of bananas, their processed products and processed consumption types is important. This will allow niche markets to be organized on an objective qualitative basis for this vastly produced crop, also for the competition of its undifferentiated commodity flows. This will, on the one hand, increase market productivity and revenue for farmers and, on the other hand, create jobs. Thus, the utility of banana fruits, peels, leaves and the potential for industrial use of these products should be explored [1].

Bananas are produced en-masse each year and some of its by-products e.g. their peel is mainly discharged as waste, while it comprises about one third of the fruit weight. The peel has historically been used for the treatment of different conditions. This by-product includes a wealth of phenolics with over 40 compounds. Phenolics are known to have good antioxidant and antimicrobial characteristics and found within banana peels, and have a variety of health benefits. However, different variables, including varieties, maturity, conditions of cultivation, and pretreatment, affect the composition and levels of these compounds. Therefore level of maturity stand as an important factor in order to determine the usability of its by-products [2]. Results of morphological characterization indicate that the basic characteristics of each banana cultivar are related to their genomes. To provide more comprehensive information on nutrient values, the study of other important vitamins and minerals was required. The banana reproduction program in Indonesia is assisted by further studies of nutrient analysis of other Indonesian banana varieties followed by selection [3]. Bananas and plantains, as interesting sources of bioactive and secondary metabolites, are commonly consumed as main source of food and for medical purposes. Bananas and plantains are of the Musa genus and are classified according to their genomic group, subgroups, use of the fruit and geographic distribution in four areas, according to their peculiar morphogenetic characteristics. Their cultivars are divided up into Eumusa, Rhodochlamys, Callimusa and the Australimusa [4].

The traditional well-known approach to assess the maturity or ripeness of fruit in a certain banana cultivar is by observing the colour of its peel. While in the tradition of digital images and hardware, the commonly chosen representation is the RGB colour space. An approach has been proposed to classify images using RGB with these specifications: to precisely group the surface- 
types in these images, either the training dataset is required to having all the brightness variation included or an approach to obtain colour features that can give high characterization exactness should be exercised. The study extracts diffused reflectance values as new colour characteristics to distinguish surface forms. With this method, data from the environment in form of Red, Green, Blue-Depth (RGB-D) and reflectance model is used to measure the diffused reflectance for a pixel for each colour channel of Red, Green, and Blue (RGB). A multi-class Support Vector Machine classification can be used for classifying surface types by diffused reflectance levels. Experiments are performed using a portable RGB-Depth sensor kit with an integrated light source for collecting surface data in a mock bridge maintenance environment. [5].

In human visual perception and statistical analysis, however, RGB colour space is not exact. The value of a single defined 'colour' has to be stated in three values and this created a wider possibility of 'neighbouring' colours which may not be of the similar tone at all. HSV colour space is then used to get more exact colour statistics in order to extract the aforementioned 'colour' characteristics. With texture characteristics extracted from images of colour textures, the wavelet transform and motif co-occurrence matrix for feature extraction are used in the HSV colour space. The horizontal, vertical and diagonal distributions in sub-bands of a transformed image are interpreted in accordance with the characteristics of the wavelet transformation. The motif co-occurrence matrix then derives texture features from the horizontal, vertical and diagonal sub-bands. The vector support machine (SVM) is used after extraction of the feature to learn texture classes and identify them by extracting features [6]. The HSV colour space is accordingly represented by a cylinder, where the angular value is the hue. For example, using a $30^{\circ}$ increment from zero, it will go through sequences of red, yellow, green, blue and violet in successive circular grades. The saturation values depend on the radius from centre and the highest value or brightness is on top of the cylinder. The very low or high light along with low saturation levels are not representative since they are either very dark or bright [7].

Thus, the Hue-Saturation-Value (HSV) colour space is considered suitable to determine stages of banana ripeness through the colour of its peel. The hue $(\mathrm{H})$ contains a single value for the tone of an object regardless of its tints and brightness. Therefore a dark and a bright red will have the same hue, as well as deep and pale red. This provides a good range in determining the overall tone of the peel. It also contains every possible colour tones perceptible by human eyes and its angle index points to a certain colour, for example 0 is red, and 0.16 is yellow, and so on. Saturation provides a separate value for how deep the tint of a colour is along its radius. Zero at the centre is the most pale (white) and 1 at the perimeter is the deepest as a pure dye. The height of the cylinder represents another separate 'value' (of the brightness), as if a light source is provided to light a colour. Thus, 0 at the bottom represents no light (black), and 1 at the top exposed the full colour. The visualisation of the HSV colour space is shown in Figure 1. 


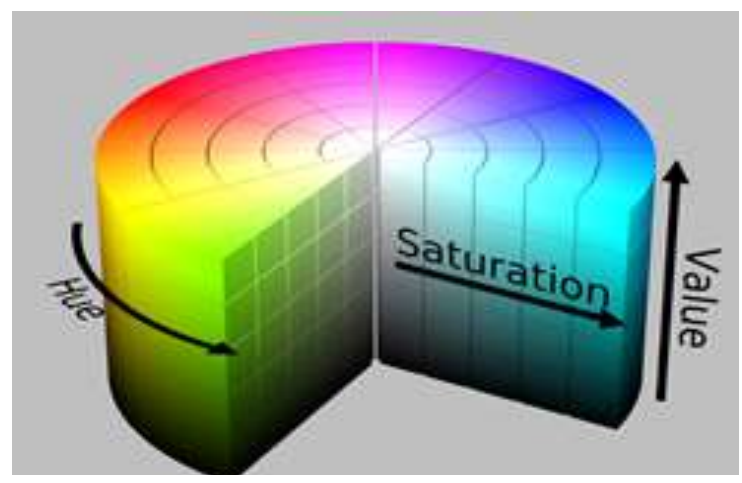

Figure 1: HSV colour space (Source: https://answers.opencv.org/questions/184281/revisions/)

\section{Methods}

The research is done in several stages which consist of preparation stage in which primary, secondary and tertiary data are collected, along with supporting tools and literatures; followed by experimental stage in which classes are determined, features extracted, along with performing training and testing using selected classification method.

\subsection{Class Determination}

Photographic images of ambon lumut, kepok and raja bananas are collected and divided into training and test data. For the training data, the features are extracted and the samples labelled according to their appropriate classes. All images are taken using the same apparatus: a Sony Cyber-Shot DC-1 pocket camera and taken under the background removed. The labels assigned to the training data are green, almost ripe, ripe and overripe.

\subsection{Feature Extraction}

HSV (Hue, Saturation, and Value) colour space is chosen due to its single-value representation of a colour tone. Thus, it allowed the colour gamut for each classes of ripeness stage to be mainly represented along a single colour line (the hue) with its tint and brightness variation, instead of a three-dimensional range using RGB (Red, Green, and Blue) colour space which might include individual unrelated colours. The range for each class is determined using the training data. Since the image obtained from the camera is in the RGB format, a conversion is performed from RGB to HSV using the following formula. The resulting pixel values now having HSV intensity, each in percentages.

The conversion consist of two determining steps, the first is to find the values of delta $(\Delta), \mathrm{Cmin}$, and Cmax as shown in Equation (1). The next is to calculate the $\mathrm{H}, \mathrm{S}$, and V values. Here $\Delta$ and Cmax play important role in which when $\Delta$ is valued zero, the $\mathrm{H}$ is directly assigned value of 0 degree. If Cmax is zero, the $\mathrm{S}$ is directly zero, and the $\mathrm{V}$ is the direct copy of Cmax, as described in Equation (2). 


$$
\begin{aligned}
& R^{\prime}=\frac{1}{255} \times R \\
& G^{\prime}=\frac{1}{255} \times G \\
& B^{\prime}=\frac{1}{255} \times B \\
& C \max =\max \left(R^{\prime}, G^{\prime}, B^{\prime}\right) \\
& C \min =\min \left(R^{\prime}, G^{\prime}, B^{\prime}\right) \\
& \Delta=C \max -C \min
\end{aligned}
$$

Hue calculation:

$$
H=\left\{\begin{array}{c}
0^{\circ}, \quad \Delta=0 \\
60^{\circ} \times\left(\frac{G^{\prime}-B^{\prime}}{\Delta} \bmod 6\right), C \max =R^{\prime} \\
60^{\circ} \times\left(\frac{B^{\prime}-R^{\prime}}{\Delta}+2\right), C \max =G^{\prime} \\
60^{\circ} \times\left(\frac{R^{\prime}-G^{\prime}}{\Delta}+4\right), C \max =B^{\prime}
\end{array}\right.
$$

Saturation calculation:

$$
S=\left\{\begin{array}{cc}
0, C \max & =0 \\
\frac{\Delta}{C \max }, C \max & \neq 0
\end{array}\right.
$$

Value calculation:

$V=C \max$

Examples of colour conversion from RGB to HSV are provided by Table 1 .

Table 1: Examples of RGB to HSV Conversion

\begin{tabular}{|l|l|l|l|}
\hline Colour & Class Examples & \multicolumn{1}{|c|}{$(\mathbf{R}, \mathbf{G}, \mathbf{B})$} & \multicolumn{1}{|c|}{$(\mathbf{H}, \mathbf{S}, \mathbf{V})$} \\
\hline & Green & $(189,174,81)$ & $\left(52^{\circ}, 57.1 \%, 74.1 \%\right)$ \\
\hline & Green & $(150,159,66)$ & $\left(66^{\circ}, 58.5 \%, 62.4 \%\right)$ \\
\hline & Green & $(174,172,85)$ & $\left(59^{\circ}, 51.1 \%, 68.2 \%\right)$ \\
\hline & Green & $(213,212,119)$ & $\left(59^{\circ}, 44.1 \%, 83.5 \%\right)$ \\
\hline & Almost ripe & $(229,215,95)$ & $\left(54^{\circ}, 58.5 \%, 89.8 \%\right)$ \\
\hline & Almost ripe & $(225,202,72)$ & $\left(51^{\circ}, 68 \%, 88.2 \%\right)$ \\
\hline & Almost ripe & $(232,211,98)$ & $\left(51^{\circ}, 57.8 \%, 91 \%\right)$ \\
\hline & Almost ripe & $(227,228,123)$ & $\left(61^{\circ}, 46.1 \%, 89.4 \%\right)$ \\
\hline & Ripe & $(232,218,108)$ & $\left(52^{\circ}, 53.4 \%, 91 \%\right)$ \\
\hline & Ripe & $(228,215,102)$ & $\left(54^{\circ}, 55.3 \%, 89.4 \%\right)$ \\
\hline & Ripe & $(212,177,69)$ & $\left(45^{\circ}, 67.5 \%, 83.1 \%\right)$ \\
\hline & Ripe & $(209,178,61)$ & $\left(47^{\circ}, 70.8 \%, 82 \%\right)$ \\
\hline & Overripe & $(211,169,91)$ & $\left(39^{\circ}, 56.9 \%, 82.7 \%\right)$ \\
\hline & Overripe & $(217,173,77)$ & $\left(41^{\circ}, 64.5 \%, 85.1 \%\right)$ \\
\hline & Overripe & $(182,142,69)$ & $\left(39^{\circ}, 62.1 \%, 71.4 \%\right)$ \\
\hline & Overripe & $(121,70,16)$ & $\left(31^{\circ}, 86.8 \%, 47.5 \%\right)$ \\
\hline
\end{tabular}




\subsection{Classification}

Labelled features of trained data (HSV images) are then stored in the database. Testing is performed by entering unlabelled images of ambon lumut, kepok and raja bananas, convert them into HSV, having their features extracted and then performed classification process. The steps for class determination are shown in Figure 2, while the Data Flow Diagrams (DFD) consisting of Context DFD, Overview DFD and Primitive DFD are shown in Figure 3 to 5.

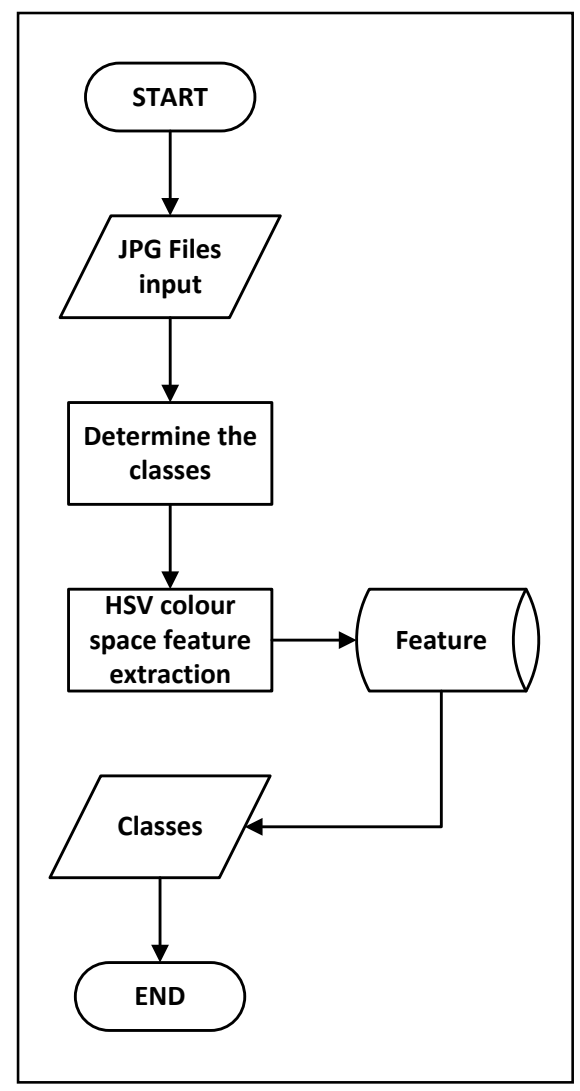

Figure 2: Class determination

Context Level 0 DFD describes the overview of the system which has a single external entity (USER). This entity provides input for the system in form of training and testing data in JPG image format. The system will then give the output of classes and the classification results, as shown in Figure 3. 


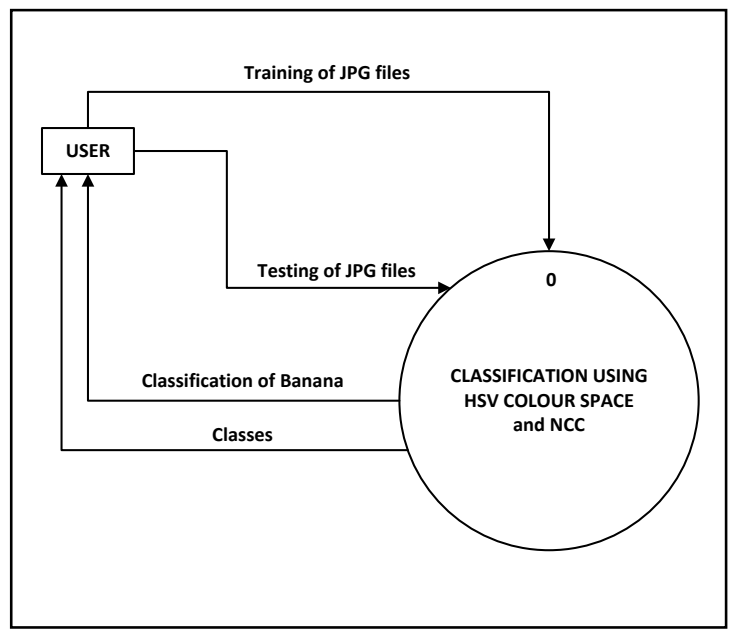

Figure 3: Context DFD of Classification Using HSV Colour Space

Breakdown of the context DFD is the Overview Diagram which describes processes exist within the system. This diagram is also called Zero Diagram or Level-0 which as shown in Figure 4, it has two processes. The first process 1.0 is the process which accepts input in the form of JPG images for training, extract its HSV colour features and store them into the Feature data store. This process also provides output in form of classes returned to external entity USER. The other process is 2.0 which is the classifier. When it is accepting input from 1.0 as a testing data, it reads the stored features from Feature data store and performs classification. The resulting classification is then returned as output to external entity USER.

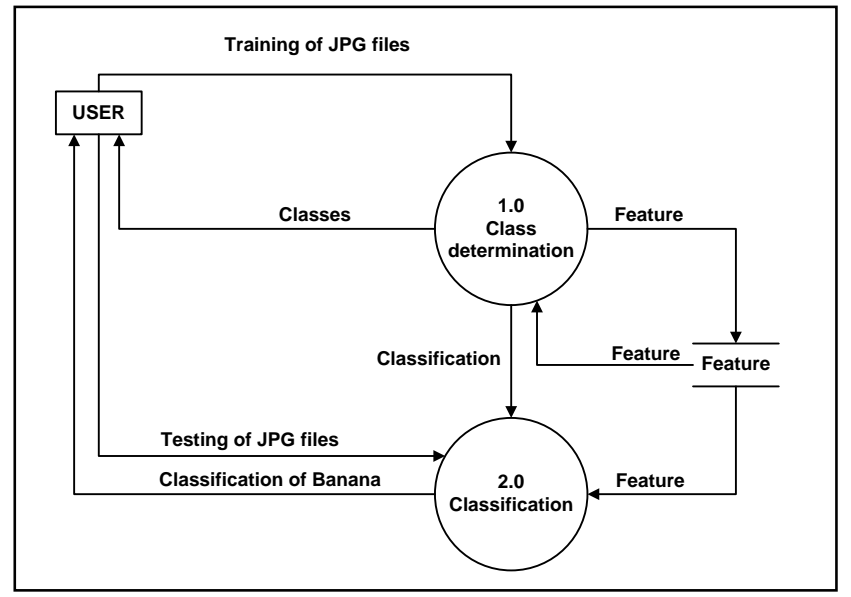

Figure 4: Overview DFD of Classification Using HSV Colour Space

The two processes can further be described using two separate diagrams of Primitive DFD or Level-1 DFD which each showed the detailed processes of Process 1 and Process 2. The breakdown of Process 1.0 are Process $1.1 \mathrm{p}$ which accepts the JPG files input, extract its features and store them to Feature data store; and Process $1.2 \mathrm{p}$ which reads the Feature data store and 
performing class determination, giving the output of classes to the external entity USER, as illustrated in Figure 5.

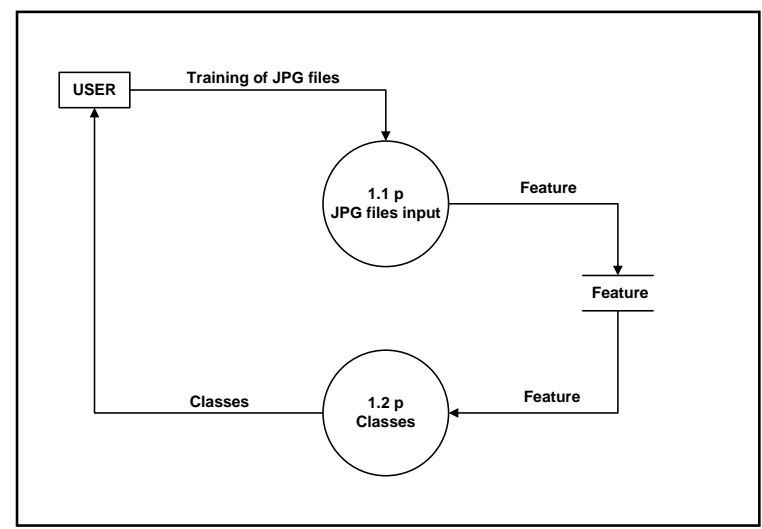

Figure 5: Primitive DFD of process 1.0 Class determination

The detailed processes of Process 2.0 is displayed in Figure 6 where Process $2.1 \mathrm{p}$ accepts the JPG file input as testing data, proceed to extract its features, then hand it over to Process $2.2 \mathrm{p}$ which performs NCC classification with already-stored features of training data from the Feature data store. The results are transferred to Process $2.3 \mathrm{p}$ which determined the class for the test data and return the classification result for the test image to the external entity USER. Therefore all the conceptual overview of the system can be completely perceived through the series of DFD Diagram.

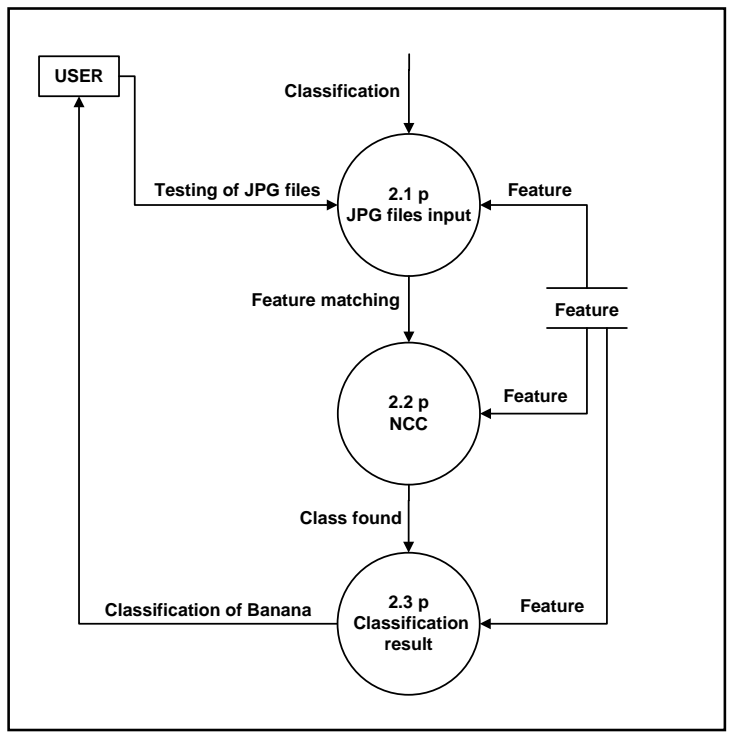

Figure 6: Primitive DFD of proses 2.0 Classification

Features from training images of all three types of banana are collected in the Feature data store and recalled to be classified against the incoming features from the test data. This research employs Nearest Centroid Classifier (NCC) to perform the classification process. In machine learning, a classification model that assigns the class label of a trained data set whose centroid is nearest to the data observed is the nearest centroid classifier or prototype classifier. Since the 
centroids indirectly represent the mean of each class, its distance from the test data can be computed to represent similarity of the data in question to the corresponding class members. Distance to the data is determined for all centroids and the smallest value is then selected. The observed data then assigned membership of the class with the nearest centroid [8].

NCC in this study employed Euclidean distance to measure the proximity of a test data to the centroids of each class, where the smallest distance belongs to the class where the test data will be assigned to. Euclidean distance d between two instances $(p, q)$ is calculated using the following formula in Equation (3), where 1..n represents the number of features. In this research, they represent each $\mathrm{H}, \mathrm{S}$ and $\mathrm{V}$ values.

Since we have four different classes, every test data undergone the distance calculation four times, each against centroids of green, almost ripe, ripe and overripe classes.

$$
\begin{aligned}
& d(p, q)=\sqrt{\left(p_{1}-q_{1}\right)^{2}+\left(p_{2}-q_{2}\right)^{2}+\cdots+\left(p_{n}-q_{n}\right)^{2}} \\
& p \quad \text { : test data } \\
& q \quad \text { : centroid of a class } \\
& p_{1 . .} p_{n} \quad \text { : features } 1 . . \mathrm{n} \text { of the test data } \\
& q_{1} . . q_{n} \quad: \text { features } 1 . . \mathrm{n} \text { of the centroid }
\end{aligned}
$$

The distribution of training data used to form the centroids of four classes (green, almost ripe, ripe, overripe) are shown in Figure 7.

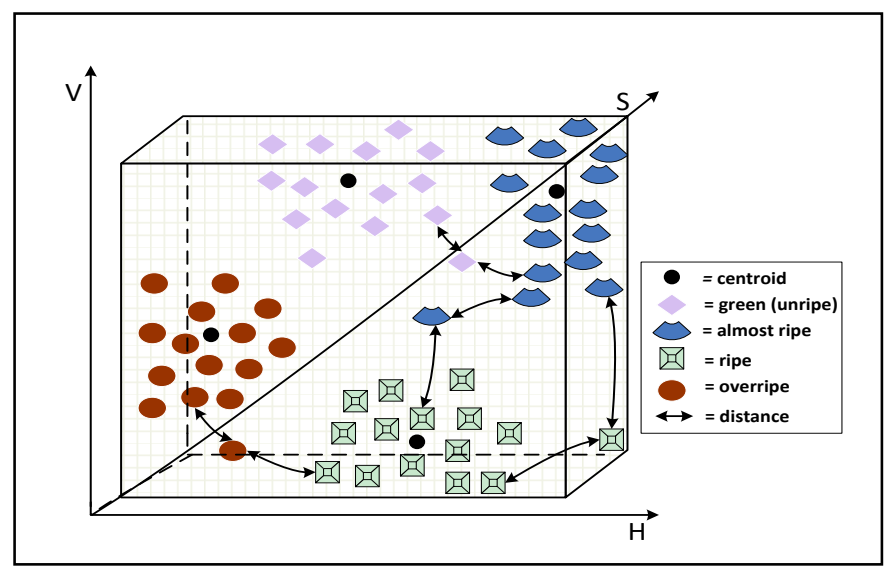

Figure 7: Data distribution in HSV colour space with NCC

The training database is formed using 60 image examples of the three kind of bananas divided into four classes (green, almost ripe, ripe and overripe). Range of features obtained for each classes are shown in Table 2.

Table 2: Class Ranges

\begin{tabular}{|l|c|c|c|c|}
\hline & $\begin{array}{c}\text { Green } \\
\text { (Unripe) }\end{array}$ & $\begin{array}{c}\text { Almost } \\
\text { Ripe }\end{array}$ & Ripe & Overripe \\
\hline $\begin{array}{l}\text { Range of } \\
\text { feature }\end{array}$ & 2151.6 & 2638.0 & 2345.2 & 2046.4 \\
\hline
\end{tabular}




\section{Result and Discussion}

Testing is performed by using 60 mixed images of ambon lumut, kepok and raja bananas: 15 green, 15 almost ripe, 15 ripe and 15 overripe, to be classified against the collected training data. Each kind of banana is not tested separately, so every image is tested against a database of mixed banana types. The classification results are presented as confusion matrix in Table 3 . The left heading is the input test data of each class, while the top heading displays the classification results.

Table 3: Confusion Matrix

\begin{tabular}{|c|c|c|c|c|}
\hline & $\begin{array}{c}\text { Green } \\
\text { (unripe) }\end{array}$ & Almost Ripe & Ripe & Overripe \\
\hline $\begin{array}{c}\text { Green } \\
\text { (unripe) }\end{array}$ & 14 & 1 & - & - \\
\hline $\begin{array}{c}\text { Almost } \\
\text { Ripe }\end{array}$ & - & 12 & - & 3 \\
\hline Ripe & - & - & 10 & 5 \\
\hline Overripe & - & - & 7 & 8 \\
\hline
\end{tabular}

From Table 3 it is clear that the green or unripe test data is the best to be classified with only one fault of 15 examples (93.33\% accuracy), while the worst is the overripe which only 8 of 15 is classified correctly (53.33\% accuracy). The second best is the almost ripe which the system correctly classified 12 out of 15 , but with 3 of them classified as overripe. This might be caused by similar colour hue of labeled 'overripe' examples of another banana type. The ripe class itself is $66.67 \%$ correctly classified ( 10 of 15 ) with acceptable mistook class of overripe.

The green examples in test data is easily classified since all three kinds of banana agreed in colour during this stage of ripeness. As the ripening progressed, each kind is showing its own characteristic of ripe colour: ambon lumut moss green or pale bright green, kepok yellow and raja yellow with a very faint orange tone. These differences explain the confusion between almost ripe, ripe and overripe classes. The research thus shows the possibility of using HSV as a feature in indicating ripeness stage of banana, albeit it is not certain whether this feature is applicable to detect the ripeness of any kind of banana using a single classification device.

\section{Conclusion}

Experiments performed on this research to test whether HSV colour features are applicable to classify ripeness stage of ambon lumut, kepok and raja banana have the following conclusions:

1. When used as colour features, HSV values are able to distinguish four classes of ripeness stage (green, almost ripe, ripe and overripe) with ripe and overripe classes shared an overlapping borderline, so that one another may wrongly classified. This may be caused by the small difference in colour tone of ripe and overripe, only differentiated by the darkness of the peel.

2. The removal of the background affects the experiment result, which increase the ability to distinguish ripe and overripe classes. 
3. Average classification rate is $73.33 \%$.

Over the findings, we have the following suggestions to improve similar future researches:

1. Although replacing the background with white improves the success rate of the classification, we suggest in employing a segmentation method over the images, so that only parts of images containing banana is in consideration for the feature extraction.

2. Employs other classification methods such as k-Nearest Neighbour (k-NN) algorithm as a comparison to Nearest Centroid Classifier (NCC) for a possibility of improvement.

3. As the colour of a starting-to-overripe banana peels includes dark freckles during the process to be entirely brown, we suggest adding a texture feature to strengthen the classification between ripe and overripe bananas.

\section{References}

[1] R. Singh, R. Kaushikand and S. Gosewade, "Bananas as underutilized fruit having huge potential as raw materials for food and non-food processing industries: A brief review," The Pharma Innovation Journal, Vol. 7 (6): pp. 574-580, May 2018, https://www.thepharmajournal.com/archives/2018/vol7issue6/PartI/7-6-103-451.pdf, accessed 10 August 2020.

[2] H.T. Vu, C.J. Scarlett and Q.V. Voung, "Phenolic compounds within banana peel and their potential uses: A review," Journal of Functional Foods, Vol. 40: pp. 238-248, January 2018, https://www.sciencedirect.com/science/article/abs/pii/S1756464617306783, accessed $10 \mathrm{Au}-$ gust 2020 .

[3] L. Hapsari and D.A. Lestari, "Fruit Characteristic And Nutrient Values Of Four Indonesian Banana Cultivars (Musa Spp.) At Different Genomic Groups," Agrivita Journal of Agricultural Science, Vol. 38 (3): pp. 303-311, May 2016.

[4] A. Pereiran and M. Maraschin, "Banana (Musa spp) from peel to pulp: Ethnopharmacology, source of bioactive compounds and its relevance for human health," Journal of Ethnopharmacology, Vol. 160: pp. 149-163, November 2014, https://www.natural-knowhow.com/wp-content/uploads/2015/07/Banana-Musa-spp-from-peel-to-pulp-Ethnopharmacology-source-ofbioactive-compounds-and-its-relevance-for-human-health-article.pdf, accessed 19 August 2020.

[5] A.W.K. To, G. Paul and D. Liu, "Surface-Type Classification Using RGB-D," IEEE Transactions on Automation Science and Engineering, Vol. 11 (2): pp. 359-366, April 2014, https://ieeexplore.iee.org/document/6675887, accessed 12 September 2020.

[6] C.D. Chang, S.S. Yu, H.H. Chen and C.S. Tsai, "HSV-based Color Texture Image Classification using Wavelet Transform and Motif Patterns," Journal of Computers, Vol. 20 (4): pp. 63-69, January 2010.

[7] D. John, "December Communication," RGB to HSV color conversion, April 2020, https://www.december.com/html/spec/colorhsltable.html, accessed 30 July 2020. 
[8] E.N. Tamatjita and A.W. Mahastama, "Comparison of Music Genre Classification Using Nearest Centroid Classifier and k-Nearest Neighbours," 2016 International Conference on Information Management and Technology (ICIMTech): pp. 118-123, May 2017, https://ieeexplore.ieee.org/document/7930314, accessed 30 July 2020. 